\title{
National Fitness 100: Significance and Operational Needs in the Context of the COVID-19 Pandemic
}

\author{
Hyun-Chul Jeong \\ Teacher, Jeonbuk National University High School, South Korea \\ 01086445918@hanmail.net
}

\begin{abstract}
The COVID-19 pandemic has led to a gradual weakening of people's health and physical strength worldwide. Therefore, it is necessary to introduce various nation-led measures to improve citizens' health. This study looked at the significance of participation in Korea's National Fitness 100 project, a national program under the National Sports Promotion Act of Korea. The study explored the project's operations to determine its significance and to evaluate its effectiveness in improving Koreans' physical fitness and well-being during the ongoing health crisis. The subjects were four fitness professionals and four citizen participants with experience with the National Physical Fitness 100 program. Data were collected through individual and group interviews. Overall, the participants expressed satisfaction with the service, saying it gave them a sense of pride and challenged them to improve. Moving forward will require the efficient operation of the Stamina 100 project and the development of additional certifications, including the Sports Activity Certification. In addition, health, nutrition, hygiene, and fitness data should be integrated through collaborative governance to monitor improvements among the participants nationwide.
\end{abstract}

Keywords: National fitness 100, Fitness certification system, Fitness certification center, Sports activity certification, COVID-19

\section{Introduction}

In 2011, the pilot project National Fitness 100 began as a national sports welfare service to maintain and promote public health. Currently, it offers free fitness tests, exercise prescription, and fitness improvement classes for people over the age of 13 across 42 regions in 16 cities and 8 areas nationwide. The project provides scientific fitness tests and indicators to measure people's physical health, but its most significant contribution is the various sports activity programs designed to help prevent infirmity and disease and make exercise and sports activities a part of Koreans' everyday lives [1]. There have longitudinal studies of similar programs in the United States, Germany, and Japan, exploring the causes and consequences of ill health over more than 50 years, and researchers have looked into ways to incorporate both aspects of the programs and the results of the studies into Korea's program [2][3]. However, previous studies on the implications of and improvements in Korea's program have focused only on the levels of participation, satisfaction, and problems with the national sports

Article history:

Received (June 2, 2020), Review Result (July 7, 2020), Accepted (August 10, 2020)

This is the proceeding of the 2020 June conference. 
welfare service. According to Korea's 2019 survey of people's participation in sports, the participation rate in daily sports (at least once a week for more than 30 minutes in a single exercise) has gradually risen from $59.2 \%$ in 2017 and $62.2 \%$ in 2018 to $66.6 \%$ in 2019 . The slow growth in participation suggests the need to provide more efficient, systematic, and engaging services to improve people's physical conditioning and strength. Thus, the National Fitness 100 project that is currently being implemented in Korea ought to be explored further.

This study examined the significance of participating in increasingly popular National Physical Fitness 100 and explored efficient operational measures to establish the project as a national sports welfare policy to maintain and promote the health of all citizens. Ideally, this study can help improve participation - and, by extension, Koreans' fitness - by suggesting ways to implement the project fully and safely during the pandemic to overcome the decline in fitness resulting from restrictions on movement and large gatherings. The study addressed the following research questions:

(1) What is the significance of participating in National Fitness 100?

(2) What can be done to ensure that the program can be run efficiently and safely during the COVID-19 pandemic?

\section{Research method}

This study used a qualitative research methodology to investigate the experiences of eight participants with intense operational experience in the National Fitness 100 project. This qualitative research method was chosen because it is a proven means of exploring and understanding the meaning that individuals or groups ascribe to a social or human problem and a form of inquiry that honors an inductive style, a focus on individual meaning, and the importance of rendering the complexity of a situation [4]. The use of semi-structured interviews made it possible to investigate the implications of and improvements in the project.

\subsection{Participants}

This study used purposeful sampling to select three exercise therapists and one physical fitness tester from fitness centers in cities $\mathrm{C}, \mathrm{D}, \mathrm{J}$, and $\mathrm{N}$, as well as four people who had experience with the project since 2017. The researcher explained the purpose of the study to participants and obtained consent before proceeding. All the participants in the study provided approval for this study to the Korea Jeonbuk National University High School. The details of the participants are shown in [Table 1].

Table 1. Participant information

\begin{tabular}{|c|c|c|c|c|c|}
\hline Number & Gender & Age & Role & Occupation & Characteristics \\
\hline 1 & M & 36 & Expert & $\begin{array}{c}\text { Exercise } \\
\text { therapist }(\mathrm{C})\end{array}$ & $\begin{array}{l}\text { Had been working as an exercise therapist at a fitness center in the C } \\
\text { area since 2016; psychology major with MPhEd }\end{array}$ \\
\hline 2 & $\mathrm{~F}$ & 32 & Expert & $\begin{array}{c}\text { Exercise } \\
\text { therapist (D) }\end{array}$ & $\begin{array}{l}\text { Had been working as an exercise therapist in D region's fitness } \\
\text { certification center since 2016; passionately developed and distributed } \\
\text { customized exercise prescription programs as part of an MPhEd project }\end{array}$ \\
\hline 3 & $\mathrm{~F}$ & 41 & Expert & $\begin{array}{c}\text { Exercise } \\
\text { therapist }(\mathrm{N})\end{array}$ & $\begin{array}{l}\text { Had been working as an exercise therapist in N region's fitness } \\
\text { certification center; physiology major with MPhEd; focused on Sports } \\
\text { Activity Certification, a recent project, and conducted much research on } \\
\text { the efficiency of the Sports Activity Certification }\end{array}$ \\
\hline 4 & $\mathrm{~F}$ & 37 & Expert & Fitness & Had been working at J region's fitness certification center since 2016 ; in \\
\hline
\end{tabular}




\begin{tabular}{|c|c|c|c|c|c|}
\hline & & & & tester $(\mathrm{J})$ & $\begin{array}{l}\text { charge of measuring fitness as an MPhEd project, guiding programs } \\
\text { linked to PAPS by school and issuing fitness certificates }\end{array}$ \\
\hline 5 & $\mathrm{~F}$ & 18 & Citizen & Student (A) & $\begin{array}{l}\text { Participated in the fitness certification system project for three years } \\
\text { (2017-2019); adopted customized programs based on exercise } \\
\text { prescriptions; steadily participated in customized program after } 2017 \\
\text { and obtained a Grade } 2 \text { certificate in } 2019\end{array}$ \\
\hline 6 & M & 40 & Citizen & $\begin{array}{l}\text { Self- } \\
\text { employed } \\
\text { (B) }\end{array}$ & $\begin{array}{l}\text { Participated in the project every three months since 2018; steadily } \\
\text { adopted programs based on exercise prescriptions; obtained a Grade } 3 \\
\text { certificate in } 2018 \text { and Grade } 1 \text { certificate in } 2019\end{array}$ \\
\hline 7 & $\mathrm{~F}$ & 53 & Citizen & $\begin{array}{c}\text { Office } \\
\text { worker }(\mathrm{C})\end{array}$ & $\begin{array}{l}\text { Participated in the project in } 2016 \text {; her fitness test showed poor fitness; } \\
\text { she reported that she tried follow the customized exercise prescription, } \\
\text { but she was too busy with work to keep up despite her willingness }\end{array}$ \\
\hline 8 & M & 68 & Citizen & Retired (D) & $\begin{array}{l}\text { First participated in the project in } 2017 \text { when he obtained a Grade } 1 \\
\text { certificate for adults; currently actively participating in the Sports } \\
\text { Activity Certification program using the app for the elderly }\end{array}$ \\
\hline
\end{tabular}

\subsection{Data collection}

The researcher collected data related on the National Fitness 100 project from March to May 2019. The in-depth interviews and group meetings with the participants were conducted from June to October 2019: the in-depth interviews were conducted four times per person (each interview lasted 30-40 minutes), and the group meetings were conducted three times (each meeting lasted 60-80 minutes). In addition, the researcher collected copies of or information about the participants' fitness certificates, exercise prescription records, sports activity apps, etc., to better understand the implementation and process of the overall project.

\subsection{Data analysis and research integrity}

To address the research problems of this study, the data were analyzed using inductive categorical analyses. Details of the contents, methods, tests, and prescriptions of the project were analyzed through individual in-depth interviews with participants and group discussions on efficient operation methods. To ensure the integrity of the in-depth interviews and the results of the group interviews, the researcher conducted three rounds of review with the members to ensure that there were no differences in the opinions of the participants. The data and contents were analyzed (multidirectional verification) by one professor of sports education and two $\mathrm{PhD}$ students in sports education to ensure the reliability and validity of the results.

\section{Significance of participating in National Fitness 100}

\subsection{Providing a variety of customized services to enhance sports welfare}

National Fitness 100 is a national sports welfare service in Korea that provides exercise counseling and prescriptions based on scientific measurements and evaluations of physical fitness conditions for the purpose of improving people's fitness and health [5]. The service provides people with free physical fitness tests and health management; the study's participants were well aware of the service, its benefits, and the expectation that people would participate.

I liked that they provide free exercise prescriptions and programs the most. Frankly speaking, it's not common for you to take care of your health and get benefits from the government as you get older. (Participant $\mathrm{D}$, individual interview) 
I really enjoyed participating in National Fitness 100. It wasn't just a fitness test, but I was provided with customized exercise prescriptions and programs while analyzing my lack of fitness. (Participant $\mathrm{C}$, individual interview)

- Provision of individual fitness assessment results and counseling

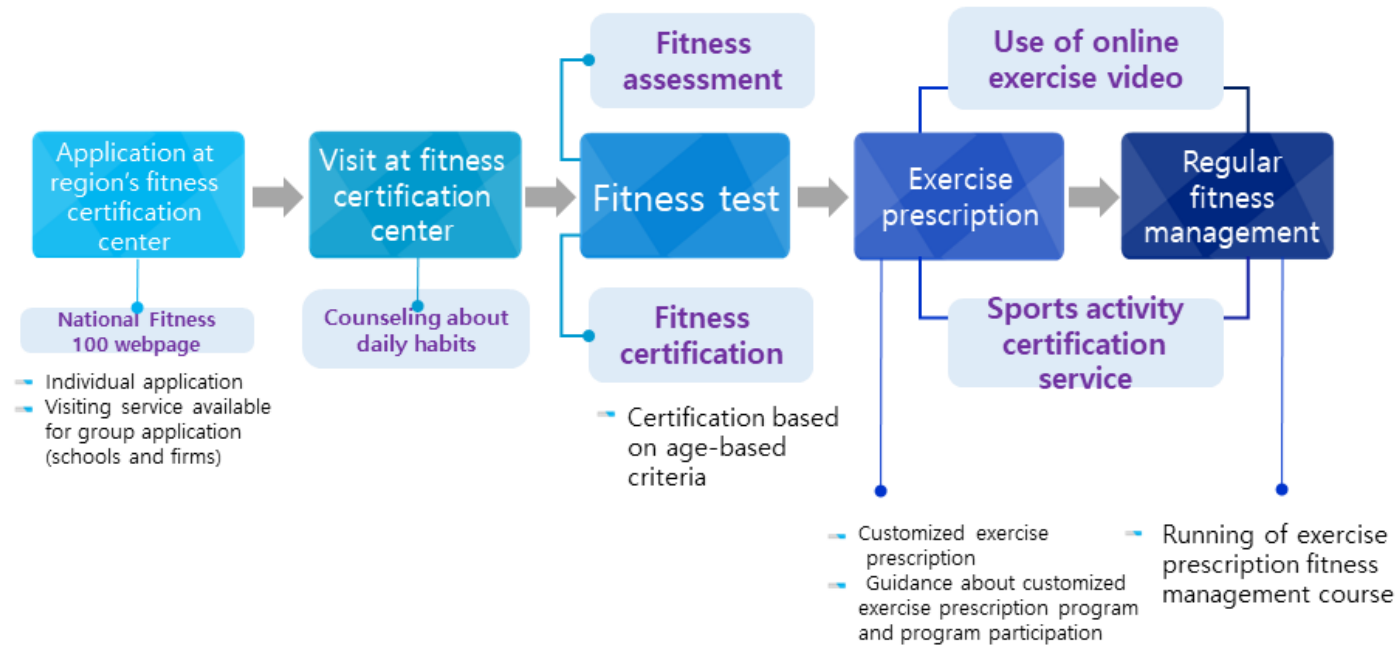

Figure 1. Fitness certification procedures for National Fitness 100

The participants reported that they were satisfied overall with the program from the fitness tests and the basic exercise program services to the customized exercise prescriptions. They affirmed that the program provided them with opportunities to become more interested in sports and to participate in sports programs more regularly. The customized exercise prescriptions helped them identify their physical fitness strengths and weaknesses, and work to improve through participation in exercise. Buck et al. found that supporting participants by identifying their strengths and praising them for their achievements helps build self-esteem [6]. The fitness test results helped people recognize their strengths, and the variety of fitness classes that are part of National Fitness 100 enabled them to focus on their improvement.

\subsection{Standardized rankings to measure progress: national fitness certificates}

The study participants believed that the biggest factor driving their participation in National Fitness 100 was the issuance of fitness certificates. All participants in the fitness tests are classified as adolescents, adults, or senior citizens; their performances during the tests are compared with the national standards for those categories, and their scores earn them an achievement ranking. Certificates of Grade 1, Grade 2, or Grade 3 achievement are then issued by the Ministry of Culture, Sports, and Tourism. The participants also receive customized personal exercise prescriptions based on their fitness test results. These results allow them to analyze their overall fitness and decide whether to participate in fitness center exercise programs or to train individually through personal exercise.

The certificate is an example of benchmarking an overseas model and applying it to our country. In fact, most of the participants in National Fitness 100 do their best to obtain the certificate. Some who fail to obtain it, work hard to make up for their weak parts and try again. (Exercise Therapist $\mathrm{C}$, individual interview) 
I have been working very hard to get a fitness certificate every year since 2017. I think the motivation for the national certification of fitness was strong. (Participant A, group interview \#1)

At first, I got a Grade 3, so I got challenged. So, I tried to improve my fitness by participating in sports programs and personally going to the gym. I was after the certificate, but as a result I was able to stay healthy while improving my physical strength. (Participant B, group interview \#1)

Kwon et al. [7] showed that the "fun factor" in the project had a positive impact on participants' intention to continue exercising. The fun factor could also be a way to challenge participants to get the best certificate grade or improve their fitness levels.

\section{Efficient operational plans for National Fitness 100}

\subsection{Extending the scope and stages of the fitness certificate}

Simple, results-based certificates are meaningful, but it is necessary to create additional incentives; one way is to systematically distribute different kinds of certificates, such as those for regularly participating in fitness-level customized exercise programs through testing and evaluation, based on the purpose of National Fitness 100.

I think the certification stages should be more diverse, and within that diversity, it should be simplified to be more complex. In other words, I personally think that the first certification should be easy, and it should get more difficult for higher certification. Because I think it is most necessary to let people have fun and a sense of fulfillment. (Participant B, individual interview)

My friend also participated to get a certificate for National Fitness 100, but he gave up on high standards as soon as possible. I felt that a certificate could be motivating but also depriving. (Exercise Therapist B, group interview \#2)

I feel that a lot, too. People come at first to try to get the certificate, but many give up because of the high standards. (Fitness Tester J, group interview \#2)

Personally, I think the diversity of certificates is needed. I think it is more important to guide people to challenge and improve individual standards in addition to general standards when prescribing exercise based on personal results. (Exercise Therapist $\mathrm{C}$, group interview \#2)

I believe that for the health and fitness of the people, it is ultimately important to have daily habits that maintain and improve fitness. So, I think we need a system for exercising habits, such as a mileage system. (Exercise Therapist N, group interview \#2)

For example, as Haag [8] showed examined the US program President's Challenge, which awards Presidential Active Lifestyle Awards according to the degree of improvement in an individual's physical fitness, and Germany's Sports Badge program. She found that there is a need to share existing individual goals with exercise therapists, in addition to providing personalized exercise programs based on exercise outcomes and to evaluating the entire process when issuing certificates.

\subsection{Improvement of sports activity certification}

The Sports Activity Certification service is a system that allows participants to receive certifications (Grades 1-3) according to their performance in sports activities in their daily lives. The program helps participants maintain and enhance their physical fitness through prescriptions based on their personal sports and exercise preferences. However, the program's 
lack of publicity and practicality - especially during the ongoing COVID-19 pandemic - have caused problems. For example, there is a need to promote the sports wearables used in sports activities and to promote efficient cases of operation.

Few people actually use the Sports Activity Certification that started in 2018. Despite the very good app and content, few people actually use them. And for the certification of sports activities, you have to go to the fitness center to print and use the evaluation sheet, but since you can't use all the wearables, it is used only for walking. In other words, the certification of sports activities was designed to encourage people to exercise more often on their own, but it is only used as a walking app. (Exercise Therapist $\mathbf{J}$, individual interview)

I got a Grade 1 certificate while participating in National Fitness 100. Then I participated in Sports Activity Certification starting last year to maintain my grade. Personally, using smart devices such as wearable devices to participate in sports, I was really happy that my activities were recorded. But few people around me participate in the Sports Activity Certification service. Fitness testing is only one-off, but Sports Activity Certification must be practiced steadily. (Participant $\mathrm{D}$, individual interview)

In addition to individual sports activities, discussions on how to certify group activities with club members are also needed, and app programs for services such as exercise prescriptions and exercise programs that are transmitted in real time should also be developed.

\subsection{Collaboration through governance activation}

In order for National Fitness 100 to become a continuous nationwide project and a benchmark for other countries, all the elements of common health fitness program should be shared and collaborated through the activation of governance to promote development in schools and communities.

Rather than PAPS, which measures physical strength at school, I think the testing conducted by National Fitness 100 is more scientific and reliable. And it's because the equipment is high-end and the satisfaction with the results is high. So, I think I'm benefiting greatly from the school and the physical fitness center's cooperation. (Participant A, individual interview)

Collaboration with schools is going well, but in fact, I think running the project in conjunction with the local health center will have much more synergy. (Exercise Therapist $\mathrm{C}$, group interview \#3)

That's right. In fact, the health promotion project conducted by the health center is largely related to National Fitness 100, and health and nutrition related content can be educated and participated in programs. (Exercise Therapist N, group interview \#3)

I think it is more efficient to prepare for a future society to carry out personalized health projects through collaboration rather than a single project. I think it is important to build various networks such as hospitals, insurance companies, and workplaces to take care of the health of the people. (Exercise Therapist J, group interview \#3)

In 2014, the Ministry of Culture, Sports, and Tourism planned a report for the Sports Bureau to reflect discounts on private insurance for those certified with excellent fitness [9]. However, there many difficulties remain due to the lack of a systematic collaboration system. For specific collaboration, individuals need to participate in common, interconnected operations through a comprehensive database of health, hygiene, territory, and fitness; in addition, national policy measures should be sought. In addition, policies specifically applicable to underprivileged areas and senior citizens are needed, as well as improvements in 
the collaboration between visiting services and National Health 100 projects linked to local health centers. The policies and programs need to be flexible enough to accommodate the measures in place to prevent the spread of the COVID-19 coronavirus.

\section{Conclusions and recommendations}

The study was conducted to examine the significance of participating in Korea's National Fitness 100 program. The main findings are as follows. There is a need to develop more efficient operation plans for the project to establish itself more widely as a national sports welfare policy to maintain and promote the health of all citizens. The participants were mostly satisfied with their participation in the sports programs, free fitness testing, and personalized exercise prescriptions, and they recognized National Fitness 100 as a statesupported sports welfare service. The state-approved fitness certificates gave participants a sense of pride and the challenge of bettering their grade motivated them to continue to participate. To improve both participation and satisfaction, the program should develop additional certifications that recognize participants' achievements of their individual goals (e.g., participation/attendance, strength/endurance improvement, etc.). The service should go beyond the existing general standards and consider processes focused on individuals' goals. The recently introduced Sports Activity Certification service should be more actively promoted to improve participation services. The operations of health, nutrition, hygiene, and fitness should be better integrated through governance for more seamless collaboration among isolated classes (including both in-person and online video-chat participation to avoid the spread of SARS-CoV-2) across regions working toward an overarching goal.

The results of this study have several implications related to operating a nation-led people's health program in the context of a pandemic. Increased isolation and inactivity, as well as restrictions on gatherings and social contact, have had a negative effect on all Koreans' health and well-being, whether or not they have been personally affected by the novel coronavirus. People need to be made aware of how they can participate in National Fitness 100 now, and they might need extra incentives to participate when fears of infection remain, such as video-conference participation certificates for the elderly and house-bound. Even once the threat has passed, the program should continue to be aware of the difficulties that prevent some people from go to the fitness centers regularly and find ways to surmount these challenges.

It is hoped that the study will help boost awareness of the project and promote efficient fitness management and participation in sports activities to improve declines in health and due to the pandemic. Further research is needed in several areas. First, individual databases should be combined into Big Data for analysis, including information from general disease management to physical fitness management. This consolidated database should be used to analyze national trends individuals' health and fitness by life cycle. Second, there should be studies focused on developing long-term strategies and plans for health and fitness policies. Medium- and long-term studies on the efficiency and effectiveness of the National Fitness 100 program should begin at once and continue for the life of the program. Third, there should be more studies on the need for diversity in exercise programs tailored to people's different circumstances (e.g., full-time workers with limited free time, the elderly and disabled, etc.). Practical research into individual programs that can be easily participated in everyday life should be carried out through the systemization of exercise programs to enhance individuals' fitness and increase participation. 


\section{References}

[1] S.-H. Jo, "Korean sport promotion Act: A series of liberal arts statutes," (2019)

[2] H.-J. Kim, "A study on the improvement in sports welfare through National Fitness 100," M.S. thesis, Korea National Sport University, Seoul, South Korea, (2018)

[3] M.-S. Kim, "Overseas cases to promote adolescent health and physical activity: US President's Challenge and the German Sports Badge," Korean Society for the Study of Physical Education, vol.18, no.2, pp.133-147, (2013)

[4] J. W. Creswell, "Qualitative inquiry \& research design: Choosing among five approaches," Sage, Thousand Oaks, pp.37, (2007)

[5] Korea Sports Promotion Foundation, National Fitness Award, http://nfa.kspo.or.kr., (2019)

[6] M. M. Buck, J. L. Lund, J. M. Harrison, and C. L. Blakemore, "Instructional strategies for secondary school physical education," McGraw-Hill College, New York, (2006)

[7] G.-I. Kwon, D.-S. Yang, and W.-W. Kim, "The effects on the psychological happiness, living satisfaction, and continuing exercise intention of the factors of fun by participating in the National Fitness 100 Program," Korean Journal of Sports Science, vol.23, no.1, pp.303-314, (2014)

[8] R. M. Haag, "A descriptive, multi-case study of physical education and physical activity within president's challenge demonstration centers," Ph.D. dissertation, Graduate School of Physical Education, Purdue University, West Lafayette, Indiana, (2013)

[9] M.-S. Kim, "National fitness award 100 in Korea," Korean Society for the Study of Physical Education, vol.19, no.1, pp.75-88, (2014)

\section{Authors}

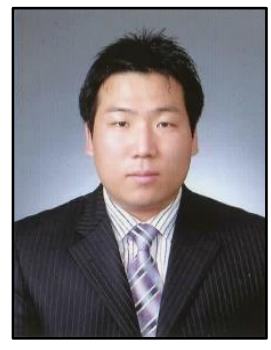

Dr. Hyun-Chul, Jeong, PhD

Physical Education teacher

Korean Association of Sport Pedagogy Innovations, Korean

Jeonbuk National University High School 